\title{
Long-term endocrine consequences of traumatic brain injury in children
}

\author{
Alan Rogol \\ Department of Pediatrics, University of Virginia, Charlottesville, Virginia, USA
}

Correspondence should be addressed to A Rogol

Email

adrogol@comcast.net
Traumatic brain injury (TBI) is a public health and socioeconomic concern worldwide and a common and serious cause of disability and death in young adults. It has been estimated that there are $90-250$ per 100,000 accidents and perhaps 100-350 per 100,000 in the general population (1). Wide variation exists in these estimates because of multiple definitions, incomplete capture of the data and inadequate standardization. Thus, these data likely underestimate the magnitude of the problem. Consequences range from physical disabilities to long-term neurocognitive, behavioral, psychological and social disruptions. It is also prevalent in infants/ children/adolescents with multiple different etiologies depending on the age group affected. The vast majority are mild (based on the Glasgow Coma Scale rating (GCS 15-12)); however, most studies note approximately $5 \%$ are severe, GCS $\leq 8$ (Coma: unconscious state, no meaningful response, no voluntary activities). Usually this portends some long-term disability. It should be noted that early fatal TBI would not be captured for any ongoing or long-term adverse events studies. The few long-term longitudinal studies indicate that TBI is becoming a chronic progressive disease enmeshed with co-morbidities as the subjects age. From the endocrine point of view, these include ongoing deficits as well as the co-morbidities of obesity and diabetes among others.

For adolescents and adults motor vehicle accidents whether as passenger or pedestrian, are the most common cause. In contrast, falls and non-accidental trauma of child abuse, especially in those younger than 6 months are more prevalent in infants. For children and adolescents sporting events cause an ever-increasing number of concussions. The latter brings up an important point concerning the longterm consequences, including pituitary function, of multiple TBIs of concussive and sub-concussive force as opposed to the single incident reported in most studies of TBI.

Potential morbidities may be both transient and permanent. Cognitive, behavioral, psychological, physiological/movement sequelae of TBI have been tracked for decades, but it is only more recently that dysregulation of hypothalamic/pituitary function has been appreciated - first in adults and then in children whether head trauma is accidental or inflicted (especially in infants). Patient selection for follow-up is made more difficult by variations in level of trauma, the hormonal system involved/evaluated and when the evaluation(s) occurred with regard to transient or more permanent dysfunction. Additionally, there are few standards or consensus guidelines for the evaluation of adults and none standardized for children and adolescents.

A few studies in adults have evaluated hypothalamic/ pituitary function within the first few days following a single traumatic event. Initially, there is the stress response noted, as in any very ill person in an intensive care unit. Decreased thyroid and gonadal axis hormone concentrations mimicking hypopituitarism are seen in a major percentage (2), but increased stress hormone concentration consisting of hyperprolactinemia, hypercortisolism and copeptin activity may also be detected. Low cortisol concentrations at this early evaluation often portend diminished hypothalamic/ pituitary function in the long term as well as being a medical emergency in the acute setting.

\section{Pathophysiology}

The primary traumatic event with its direct injury (coup) and indirect injury (countercoup) includes shearing of blood vessels and perhaps neurons (primary injury). The secondary insults: hypotension, hypoxia, increased intracranial pressure, changes in cerebral blood flow, metabolism and inflammation prolong the injury in both time and volume of brain tissue involved $(2,3$, 4). Symptoms and transient hormone deficiency may track the triphasic blood flow response: on day zero hypoperfusion, followed by cerebral hyperemia mainly on days 1-3 and ending with variably low perfusion due to

Published by Bioscientifica Ltd. 
vasospasm. This pattern has been noted in severe TBI, but not well studied in TBI of lesser magnitude. This pattern is considered more likely based upon autopsy studies from victims studied soon after the trauma in which hemorrhagic changes were found in the hypothalamus and pituitary.

Where studied, the spreading proinflammatory response was not well correlated to the GCS number at the time of injury. The hypothalamus and anterior pituitary are vulnerable especially the latter which is supplied by the long hypophyseal vessels and the portal capillaries of the pituitary stalk. Growth hormone deficiency has been considered the most common single pituitary deficiency in most studies beyond the acute period and is likely due to the somatotropes being mainly laterally situated, especially in relationship to the more centrally located thyrotropes and corticotropes, and thus, further away from the feeding vasculature. More recently, an autoimmune mechanism causing hypopituitarism following head trauma has been considered, but not yet shown conclusively (5). However, there are intriguing data from combat sports whose participants have multiple relatively low-impact head injuries showing a correlation between number of bouts, autoimmunity and hypopituitarism.

The responses to repetitive, low severity head injury as might be noted in athletic endeavors is understudied. In former symptomatic professional American football players who often suffer multiple concussions and hundreds of sub-concussive blows to the head, the incidence of hypopituitarism approaches $20 \%$ and that for metabolic syndrome, $50 \%$. What was not noted was the interval between concussions (or sub-concussive head trauma), which is important because of the metabolic vulnerability to a second injury before complete recovery from the first has had time to occur.

Early in the course of recovery from an episode of TBI one must consider the physiologic response to acute stress (upregulation of the HPA axis and hyperprolactinemia), which is especially important with regard to the corticotropes, secondary adrenal insufficiency and the $\mathrm{ADH}$ neurons. It is during this time that prevention of secondary injury is so critical, requiring special care to lessen hypoxia and hypotension. Early hypocortisolemia is strongly associated with chronic hypopituitarism, whereas central hypothyroidism and hypogonadism are not.

The time after the initial insult is an important factor in evaluating the endocrine consequences of injury with a number of transient hormonal deficiencies; however, transient secondary adrenal insufficiency should be considered. Although there are a number of deficits in hypothalamic-pituitary function in the first few months, not all persist to a year or more, although it seems that if present at 1 year, the deficit is more likely to be permanent. There is an apparent overlap between longterm symptoms after TBI and chronic hypopituitarism with relevance to the diagnosis of the latter and chronic replacement therapy to reverse diminished linear growth and decreased quality of life, and unfavorable body composition, lipid profile, BMI and waist circumference.

Radiation therapy in children for brain tumors or leukemia may be considered another form of traumatic brain injury. The longer term responses to it may inform about anterior pituitary hormone deficiencies, and in young children, precocious puberty, as is a focus of the highlighted article (6). The effects of the treatment are localized, the dose quantitated and the timing and dose distribution (fractionation) known. Precocious puberty and even rapidly progressive pubertal maturation beginning within the normal age range have occurred with doses to the hypothalamus of $\geq 18$ Gy especially in girls less than 4 years old. It should be noted that many of the patients in these trials and those with TBI would not be at risk for precocious puberty based on their age, and it is often difficult to determine an abnormally rapid pubertal trajectory from the broad range of normal. However, most of the hypothalamic/pituitary deficits noted in those receiving radiation therapy evolve slowly (over years) and are likely permanent. Those characteristics make the study design of Crosnier and coworkers, so important for their study encompassed 5 or more years in most of their patients (6). For children an example is the presence of growth failure and short stature due to GH deficiency. In such children the pre-test probability of GH deficiency is high and quite appropriate to test, rather than testing everyone, especially those with a very low pre-test probability of being GH deficient.

\section{Severe TBI in children}

\section{Growth}

Crosnier and colleagues note the consequences of severe TBI in infants, children and adolescents. They appropriately note that this group represents a small fraction of those who suffer TBI and focus on the neuroendocrine aspects of the traumatic injury, but more importantly on the longer term aspects. In their earlier publication (7) they note follow-up until 1 year when 
approximately $1 / 3$ had pituitary dysfunction, mainly GH deficiency. Their goal in the present work (6) is to detect the development of other pituitary dysfunction, for example, growth disorders or abnormalities of pubertal maturation, as well as to determine if the 'original' deficits remained. The patient population was strictly defined as being $<15$ years old at initial incident which was considered severe based on Glasgow Coma Scale as $\leq 8$, representing only approximately $5 \%$ of all TBI in this age group. Their investigations included standardized tests for anterior pituitary function, including the end-organ hormones for thyroid and adrenal, prolactin levels, especially as it reflects an intact pituitary stalk and some standardized tests of GH function. It should be noted that sex hormone priming was not done for any of the children, irrespective of their pubertal status, that obesity as indexed by the BMI was not considered in the responses, despite it being a well-known inhibitor of $\mathrm{GH}$ release and overnight spontaneous GH release considered without reference to normal values. It should be noted that spontaneous overnight or 24-h release of GH may vary multiple-fold in normally growing children whether pre- or pubertal (8). The situation brings up the issue of pre-test (prior in the Bayesian sense) probability of an abnormally low GH response to any stimulus. The chance of a biologically important deficiency of GH is exceedingly small in a child who is growing normally, has a bone age within normal limits and an IGF-1 SDS above 0. Some of the patients who tested as GH deficient at 1 year were considered 'transient' because they 'recovered' at final testing when their clinical phenotype was noted to be normal height velocity, attained height and pubertal maturation. I suspect that that has more to do with testing in a group of children/adolescents with low prior probability of accurate results with imperfect tests.

What if GH deficiency is confirmed? It is known that GH deficiency (of any cause) in the adult can affect quality of life (QoL) when determined by objective criteria. Thus, QoL is an important goal of therapy. GH deficiency may interfere with some cognitive, behavioral and physical rehabilitation of children following severe TBI.

\section{Pubertal maturation}

Pubertal maturation is uncommonly affected by the long term consequences of TBI. The present authors note that 4 of 61 patients with long-term follow-up from severe TBI had precocious puberty when diagnosed by the usual criteria. It is important to note that not all 61 were under the age for normal pubertal maturation nor did some have a significant interval between the injury and the usual time for puberty in the general population. Data included in their Table 3 show that all were within one year of early normal puberty ( 3 females over age 7 ) and the lone boy was already 9 . All began puberty between 2 and 6 years after the accident and had very increased height velocity and LH responses to GnRH well into the pubertal range except for one within the lowest range. Two had very increased BMI, another factor consistent with earlier pubertal maturation, but considered precocious by the standard definition. Others have shown a few with precocious puberty following TBI but in general show a rapid tempo of pubertal maturation following severe TBI with a variable interval between the trauma and the physical alterations of pubertal maturation.

\section{Dénouement}

Traumatic brain injury is an increasingly important issue for acute care, but also because it often becomes a series of chronic health issues: physical/movement, cognitive, behavioral and psychosocial. The cost to society is great and it may lead to long-term diminished quality of life. The endocrine-related aspects, particularly hypothalamicpituitary-end organ axes, have become better known and studied over the past two decades. Relevant issues include severity of the inciting episode, most commonly indexed by the Glasgow Coma Scale and grouped as mild, moderate or severe for most analyses. The existing literature indicates widely variable incidence of the disordered hypothalamicpituitary-end organ axes, depending on when after the incident the evaluation is performed. An acute evaluation may overlap with the effects serious illness, such as noted in intensive care units. At that time the most important endocrine system finding is adrenal insufficiency at a time when the physiologic response should be upregulation of copeptin and cortisol. Clearly replacement at high stress dose levels is indicated. The acute response may indicate euthyroid sick or central hypogonadism and hypothyroidism with hyperprolactinemia. None of these acute and often transient abnormalities require therapy in the short time frame.

After a few weeks to months some axes recover and others may still show transient dysfunction. By a year's time most findings indicate permanent hypopituitarism with some very important caveats: Growth hormone testing may have abnormal results in approximately $1 / 3$ of patients, but most not permanently GH deficient given that the pre-test probability is low and they have no symptoms compatible with GH deficiency. It is clearly 
easier in children who have growth potential - it really does not matter what the result is (or whether the test should have been done) in a child with normal height velocity, bone age within a year of chronological age and an IGF-1 level above the mean. Many of those noted are obese which confounds usual GH testing. For adults quality of life based on one of the many inventories and changing body composition may indicate who should be tested; however, a number of neurocognitive symptoms post TBI overlap with adult GH deficiency.

Pubertal alterations are not as common as those for growth in children/adolescents. Pathologically delayed puberty because of permanent hypogonadotropic hypogonadism is uncommon. More likely are the alterations of advanced pubertal maturation whether discovered as central precocious puberty or rapid progression (tempo) of an early normally timed pubertal maturation. Again there are confounders and the case series published of ages very close to the early, normal start of puberty and significant obesity as indexed by BMI SDS. One cannot dismiss patients with true precocious puberty, but not all would escape the leading 'tail of Gauss' for the timing of the start of pubertal maturation.

How do all of these concepts help us? The pediatric endocrinologist may not be involved in the acute care of a child with a severe TBI. It is usually the intensivists, neurologists and neurosurgeons who evaluate acutely and likely the neurologists, and developmental pediatricians along with the child's primary care physician who provide the long term care and evaluations. I believe it incumbent upon us as pediatric endocrinologists to educate the parents of the children, the primary care physicians, the neurologists and the developmental pediatricians-the latter are often aware of growth and puberty. We should teach about when such children might be evaluated by a pediatric endocrinologist to diagnose early disorders of the hypothalamic-pituitary-adrenal, gonadal and growth axes to diagnose insufficiencies (and precocious puberty) and prevent problems such as precocious early maturation in someone with marginal GH sufficiency who would grow at a normal rate but at the expense of a rapidly progressing bone age and (preventable) short adult stature.

Strengths of this study include the relatively large sample size, homogeneity of the severity of the TBI sustained by the subjects and the long follow-up interval. There are several important take-home points. One is that significant endocrinopathies can evolve over time requiring surveillance of indefinite duration in children who have experienced a TBI. Another is that short insults to endocrine systems may be transient, reflecting the dynamic nature of physiology in the setting of acute injury. Finally, this report will hopefully raise awareness of potential endocrine sequelae of TBI among those who on the front lines of caring for affected children and adolescents.

\section{Declaration of interest}

The author declares that there is no conflict of interest that could be perceived as prejudicing the impartiality of this commentary.

\section{Funding}

This research did not receive any specific grant from any funding agency in the public, commercial or not-for-profit sector.

\section{References}

1 Menon DK, Schwab K, Wright DW \& Maas AI. Position statement: definition of traumatic brain injury. Archives of Physical Medicine and Rehabilitation 201091 1634-1640.

2 Klose M \& Feldt-Rasmussen U. Chronic endocrine consequences of traumatic brain injury - what is the evidence? Nature Reviews Endocrinology 201814 57-62. (https://doi.org/10.1038/ nrendo.2017.103)

3 Dusick JR, Wang C, Cohan P, Swerdloff R \& Kelly DF. Pathophysiology of hypopituitarism in the setting of brain injury. Pituitary 201215 2-9. (https://doi.org/10.1007/s11102-008-0130-6)

4 Salehi F, Kovacs K, Scheithauer BW, Pfeifer EA \& Cusimano M. Histologic study of the human pituitary gland in acute traumatic brain injury. Brain Injury 200721 651-656. (https://doi. org/10.1080/02699050701426956)

5 De Bellis A, Bellastella G, Mallorino MI, Costatino A, Cirillo P, Longo M, Pernice V, Bellastella A \& Esposito K. The role of autoimmunity in pituitary dysfunction due to traumatic brain injury. Pituitary 2019 Epub. (https://doi.org/10.1007/s11102-019-00953-z)

6 Dassa Y, Crosnier H, Chevignard M, Viaud M, Personnier C, Fletchner I, Meyer P, Puget S, Boddaert N, Breton S et al. Pituitary deficiency and precocious puberty after childhood severe traumatic brain injury: a long-term follow-up prospective study. European Journal of Endocrinology 2019180 281-290. (https://doi.org/10.1530/ EJE-19-0034)

7 Personnier C, Crosnier H, Meyer P, Chevignard M, Flechtner I, Boddaert N, Breton S, Mignot C, Dassa Y, Souberbielle JC et al. Prevalence of pituitary dysfunction after severe traumatic brain injury in children and adolescents: a large prospective study. Journal of Clinical Endocrinology and Metabolism 201499 2052-2060. (https:// doi.org/10.1210/jc.2013-4129)

8 Martha PM, Jr, Rogol AD, Veldhuis JD, Kerrigan JR, Goodman DW \& Blizzard RM. Alterations in the pulsatile properties of circulating GH concentrations during puberty in boys. Journal of Clinical Endocrinology and Metabolism 198969 563-570. (https://doi. org/10.1210/jcem-69-3-563) 